\title{
Ionospheric Scattering Effects in Long-Distance Propagation
}

\author{
H. A. Whale
}

\author{
Contribution from Seagrove Radio Research Station, The University of Auckland, New Zealand \\ (Received December 7, 1962)
}

\begin{abstract}
A statistical theory of the behavior of rays scattered at successive ionospheric reflections in propagating around a spherical earth is given. The theory predicts the properties of the antipodal area, the deviation of bearing to be expected at places off the main transmitting beam and the spread of the incoming fan of rays at all distances. Special properties appear to be associated with reception at an angular distance of about $255^{\circ}$ from the transmitter. The effects of an absorbing region on the received direction and spread of rays are discussed.
\end{abstract}

\section{Introduction}

In two previous papers [Whale, 1959; Whale, 1962] the effects of scattering by the ionosphere in long-distance shortwave propagation have been discussed. In the former paper the concepts of small-circle propagation and the antipodal area were introduced; in the latter a statistical treatment of the scattering problem for a flat earth was presented. In the present paper, this treatment is modified to cover the case of a spherical earth and thus provides solutions to many of the problems which were treated less satisfactorily previously. In particular, relations are derived for the mean bearing deviation to be expected when signals are received from a beamed transmitting antenna aimed in some other direction.

Many of the effects predicted by this theory have been observed experimentally. These experimental results will be described in detail in a further paper.

The assumption is made that the ionosphere is a spherical reflecting and scattering surface concentric with the surface of the earth. In practice, this is not quite true; the eccentricity of the effective reflecting surface of the ionosphere with respect to the earth's surface leads to two effects. The first of these is a shift of the position of the antipodal area away from the point geometrically antipodal to the transmitter. The second, and less obvious effect relates to bearing deviations at places about $255^{\circ}$ angular distance from the transmitter. At this distance, the theory predicts that, under conditions of spherical symmetry and uniform transmissibility of the ionosphere, the mean signal direction should be along the great circle irrespective of the sharpness or direction of the transmitter beam or of the value of the ionospheric scattering factor.

In the last section, the effects of an absorbing region on the received signal are discussed.

\section{Omnidirectional Transmitting Antenna}

Consider an omnidirectional transmitting antenna located at $T$ in figure 1 , where the great circle to the receiver is $T-R$. Let $T U, U V, V W$ be successive traces of the ray path on the surface of the earth so that $T U$ is from the ground to the ionosphere, $U V$ is from the ionosphere back to the ground and so on. We will assume that the overall effect of scattering at the ionosphere and at the ground can be represented by an average effect of the same magnitude at all reflections, whether at the ground or the ionosphere. Let $T U=U V=V W=$ 


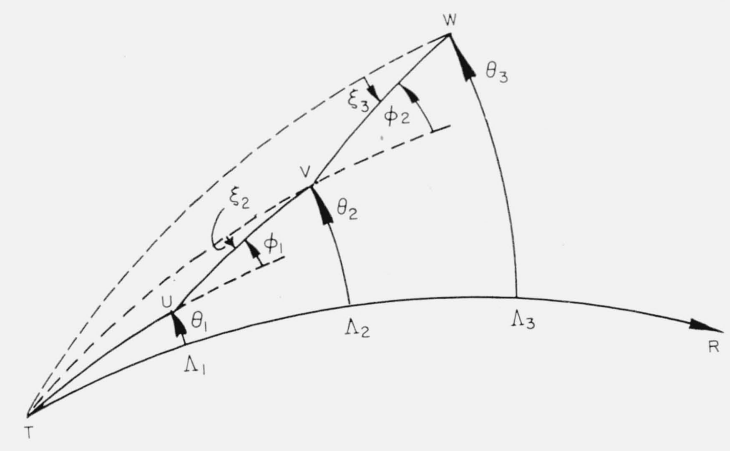

FIGURE 1. Scattering at successive reflection lines $\Lambda_{1}, \Lambda_{2}, \ldots$

. . . $=\lambda$, where all distances are measured as angles subtended at the earth's center. Let the scattering at each reflection be described by the scattering function

$$
P(\phi) \propto \exp -A \phi^{2},
$$

i.e., an incident plane wave in the direction $\phi=0$ becomes a fan of waves, the power in the directions contained between the angles $\phi$ and $\phi+d \phi$ being given by (1). The spread of this fan of waves can be specified either by the standard deviation, $\sigma$, where

$$
\sigma=\frac{1}{\sqrt{2 A}}=\frac{0.707}{\sqrt{A}}
$$

or by the angle between the direction of the maximum $(\phi=0)$ and the half-power point given by

Thus

$$
\sigma^{\prime}=\frac{0.832}{\sqrt{A}} .
$$

$$
\sigma^{\prime}=1.18 \sigma, \text { or the beam width }=2 \sigma^{\prime}=2.35 \sigma \text {. }
$$

We can now proceed as follows. Let the primed functions refer to the rays incident at one of the scattering reflections and the unprimed functions refer to the emergent rays. The power distribution along the line $\Lambda_{1}$ is given by

and

$$
P\left(\Lambda_{1}\right) \propto \exp -A \phi_{1}^{2}
$$

$$
P^{\prime}\left(\Lambda_{2}\right) \propto \exp -\frac{A S_{2}^{2}}{S^{2}} \xi_{2}^{2}
$$

since

where

$$
\frac{\sin \phi_{1}}{S_{2}}=\frac{\sin \xi_{2}}{S} \text { i.e., } \phi_{1} \approx \frac{S_{2}}{S} \xi_{2}\left(\phi_{1}, \xi_{2} \text { are small }\right),
$$

Then,

$$
S_{2}=\sin \Lambda_{2}, S=\sin \lambda .
$$

$$
\begin{gathered}
P\left(\Lambda_{2}\right) \propto \int_{-\infty}^{\infty} P^{\prime}\left(\Lambda_{2}\right) \cdot \exp -A\left(\phi_{2}-\xi_{2}\right)^{2} \cdot d \xi_{2} \\
=\exp -\frac{A S_{2}^{2}}{S^{2}+S_{2}^{2}} \phi_{2}^{2} .
\end{gathered}
$$

Now,

Therefore

$$
\frac{\sin \phi_{2}}{S_{3}}=\frac{\sin \xi_{3}}{S_{2}} \text { i.e. } \phi_{2} \approx \frac{S_{3}}{S_{2}} \xi_{3} \text { since } \phi_{2}, \xi_{3} \text { are small. }
$$

$$
P^{\prime}\left(\Lambda_{3}\right) \propto \exp -A \frac{S_{3}^{2}}{S^{2}+S_{2}^{2}} \xi_{3}^{2} .
$$


Performing the same integration on $P^{\prime}\left(\Lambda_{3}\right)$ as in (4), we have

The general forms are thus:

$$
P\left(\Lambda_{3}\right) \propto \exp -A \frac{S_{3}^{2}}{S^{2}+S_{2}^{2}+S_{3}^{2}} \phi_{3}^{2} .
$$

$$
\begin{gathered}
P^{\prime}\left(\Lambda_{n}\right) \propto \exp -A \frac{S_{n}^{2}}{S^{2}+S_{2}^{2}+S_{3}^{2}+\ldots+S_{n-1}^{2}} \xi_{n}^{2} . \\
P\left(\Lambda_{n}\right) \propto \exp -A \frac{S_{n}^{2}}{S^{2}+S_{2}^{2}+S_{3}^{2}+\ldots+S_{n}^{2}} \phi_{n}^{2} .
\end{gathered}
$$

The denominators are relatively slowly varying functions so that we may use the approximation:

$$
S_{n}=\sin \Lambda_{n} \approx \sin n \lambda \text {. }
$$

In any actual case, the angular distance from the transmitter to the receiver is known $\left(T R=\Lambda_{n}\right)$ and $\lambda$ must be deduced from the number of hops (either assumed or found from measurements of vertical angles).

The series in the denominators of (8) and (9) can be summed to yield:

$$
\begin{gathered}
P^{\prime}\left(\Lambda_{n}\right) \propto \exp -\frac{2 A \sin \lambda \cdot \sin ^{2} \Lambda_{n}}{(n-1) \sin \lambda-\cos \Lambda_{n} \sin \left(\Lambda_{n}-\lambda\right)} \xi_{n}^{2} \\
P\left(\Lambda_{n}\right) \propto \exp -\frac{2 A \sin \lambda \sin ^{2} \Lambda_{n}}{n \sin \lambda-\cos \left(\Lambda_{n}+\lambda\right) \cdot \sin \Lambda_{n}} \phi_{n}^{2} .
\end{gathered}
$$

In a previous publication, an approximate value for the spread of a wave received at a distant point was obtained using a theory based on small circle propagation [Whale, 1959]. It is interesting to compare this former result with the one obtained above.

The standard deviation of the spread calculated from (10) can be written as:

$$
\sigma=\frac{1}{\sqrt{2 K}} \text { where } K=\frac{2 A \sin \lambda \sin ^{2} \Lambda_{n}}{(n-1) \sin \lambda-\cos \Lambda_{n} \sin \left(\Lambda_{n}-\lambda\right)}
$$

For comparison, put $\lambda=9^{\circ}$, and $A$ such that $\sigma=2^{\circ}$ at $\Lambda_{n}=90^{\circ}$, i.e., $A \approx 2000$, in this case. The small circle formula becomes

$$
\text { (sin) } \Delta \theta=\sin \sigma=0.035 \tan \frac{\Lambda_{n}}{2} .
$$

The two curves are plotted in figure 2. There are significant differences between the two. The statistical theory given above is useful up to any distance, even beyond the antipodal point, while the previous theory could not be extended to that distance.

\section{Directional Transmitting Antenna}

The directivity of the transmitting antenna may be introduced as a term involving $\theta$ (fig. 1). If this directivity is of the form

then

$$
P(\theta) \propto \exp -B \theta^{2},
$$

$$
P\left(\Lambda_{1}\right) \propto \exp -B \theta_{1}^{2} \cdot \exp -A \phi_{1}^{2}
$$

where the point at which the observations are made is specified in terms of the coordinates $\left(\Lambda_{1}, \theta_{1}\right)$ i.e., colatitude and longitude measured from the center of the antenna beam.

If we proceed as in section 2, the expressions for the distribution of directions at greater distances rapidly become very complicated. The general expressions are given below. If the 


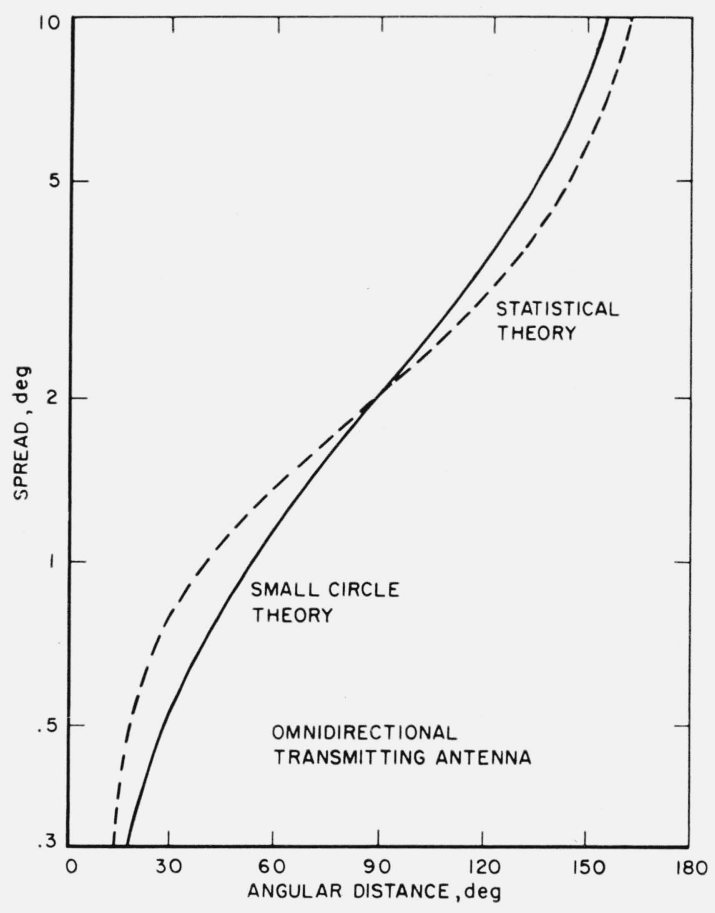

FIGURE 2. Comparison of the spread derived from the earlier small circle theory with that from the present theory.

distributions along successive lines are required, it is more economical of time to compute each distribution from the previous one using the recurrence relations. If the expression for $P^{\prime}\left(\Lambda_{n}\right)$ is given by

then

$$
P^{\prime}\left(\Lambda_{n}\right) \propto \exp -\left(L^{\prime} \theta_{n}^{2}-M^{\prime} \theta_{n} \xi_{n}+N^{\prime} \xi_{n}^{2}\right)
$$

$$
P\left(\Lambda_{n}\right) \propto \exp -\left\{\left[L^{\prime}-\frac{M^{\prime 2}}{4\left(A+N^{\prime}\right)}\right] \theta_{n}^{2}-\frac{A M^{\prime}}{A+N^{\prime}} \theta_{n} \phi_{n}+\frac{A N^{\prime}}{A+N^{\prime}} \phi_{n}^{2}\right\} .
$$

If this is now writteil

then

$$
P\left(\Lambda_{n}\right) \propto \exp -\left(L \theta_{n}^{2}-M \theta_{n} \phi_{n}+N \phi_{n}^{2}\right),
$$

$P^{\prime}\left(\Lambda_{n+1}\right) \propto \exp -\left\{L \theta_{n+1}^{2}-\left[2 L \frac{S}{S_{n}}+M \frac{S_{n+1}}{S_{n}}\right] \theta_{n+1} \xi_{n+1}\right.$

where

$$
\left.+\left[L\left(\frac{S}{S_{n}}\right)^{2}+M\left(\frac{S}{S_{n}}\right)\left(\frac{S_{n+1}}{S_{n}}\right)+N\left(\frac{S_{n+1}}{S_{n}}\right)^{2}\right] \xi_{n+1}^{2}\right\}
$$

$$
S=\sin \lambda . \quad S_{n}=\sin n \lambda .
$$

The general forms of the expressions for the probability distributions may be written as follows:

If $P\left(\Lambda_{n}\right) \propto \exp -\left(L \theta_{n}^{2}-M \theta_{n} \phi_{n}+N \phi_{n}^{2}\right)$

then

$$
\begin{aligned}
L & =\frac{A B}{A+B \frac{(n-1) S^{2}+(n-2) S_{2}^{2}+\ldots S_{n-1}^{2}}{S^{2}+S_{2}^{2}+\ldots+S_{n}^{2}}} \\
& =\frac{A B}{D} .
\end{aligned}
$$




$$
\begin{aligned}
& M=\frac{A B}{D} \cdot\left\{\frac{S_{n-1}}{S}-\frac{S_{n+1}}{S} \cdot \frac{S^{2}+S_{2}^{2}+\ldots S_{n-1}^{2}}{S^{2}+S_{2}^{2}+\ldots S_{n}^{2}}\right\}, \\
& N=\frac{A}{D} \cdot\left\{B+A \frac{S_{n}^{2}}{S^{2}+S_{2}^{2}+\ldots S_{n-1}^{2}}\right\} \cdot\left\{\frac{S^{2}+S_{2}^{2}+\ldots S_{n-1}^{2}}{S^{2}+S_{2}^{2}+\ldots S_{n}^{2}}\right\} .
\end{aligned}
$$

Similarly, if $P^{\prime}\left(\Lambda_{n}\right) \propto \exp -\left(L^{\prime} \theta_{n}^{2}-M^{\prime} \theta_{n} \xi_{n}+N^{\prime} \xi_{n}^{2}\right)$,

then

$$
\begin{aligned}
L^{\prime} & =\frac{A B}{A+B \frac{(n-2) S^{2}+(n-3) S_{2}^{2}+\ldots+S_{n-2}^{2}}{S^{2}+S_{2}^{2}+\ldots+S_{n-1}^{2}}} \\
& =\frac{A B}{D^{\prime}}, \\
M^{\prime} & =\frac{A B}{D^{\prime}} \cdot\left\{2 \frac{S}{S_{n-1}}+\left(\frac{S_{n-2}}{S}-\frac{S_{n}}{S} \cdot \frac{S^{2}+S_{2}^{2}+\ldots S_{n-2}^{2}}{S^{2}+S_{2}^{2}+\ldots S_{n-1}^{2}}\right) \frac{S_{n}}{S_{n-1}}\right\}, \\
N^{\prime} & =\frac{A}{D^{\prime}} \cdot\left\{B+A \frac{S_{n}^{2}}{S^{2}+S_{2}^{2}+\ldots S_{n-1}^{2}}\right\} .
\end{aligned}
$$

The series appearing in (19) to (25) may be summed but the relations between the various quantities can be seen rather more readily when they are written in the above forms.

At any place specified by the coordinates $\left(\theta_{n}, \Lambda_{n}\right)$ the spread of the incoming wave may be found directly from the expression for $P^{\prime}\left(\Lambda_{n}\right)$. On the antenna axis (i.e., in the direction of the main beam) $\theta_{n}=0$, so that

$$
P^{\prime}\left(\Lambda_{n}\right) \propto \exp -N^{\prime} \xi_{n}^{2}
$$

The spread of the directions is thus given by

$$
\sigma=\frac{1}{\sqrt{2 N^{\prime}}} \cdot
$$

At places off the axis of the main beam, the received signals are no longer symmetrically spread about the great circle direction to the transmitter. The direction from which maximum signals are received is given by $d P^{\prime} / d \xi=0$ i.e.,

$$
\xi=\frac{M^{\prime} \theta_{n}}{2 N^{\prime}}
$$

To obtain the polar diagram of the transmitting antenna as measured with an omnidirectional antenna at the receiving site, the total power received as $\theta$ is varied is found from $\int_{-\infty}^{\infty} P^{\prime} d \xi$ i.e.,

$$
P(\theta) \propto \exp -\left(L^{\prime}-\frac{M^{\prime 2}}{4 N^{\prime}}\right) \theta^{2}
$$

\section{Spread of Incoming Directions}

The directions of the signals arriving at any place with coordinates $(\Lambda, \theta)$ are contained in the expression

$$
P^{\prime}(\Lambda) \propto \exp -\left(L^{\prime} \theta^{2}-M^{\prime} \theta \xi+N^{\prime} \xi^{2}\right) .
$$

On the antenna axis $\theta=0$, so that $\sigma=\frac{1}{\sqrt{2 N^{\prime}}}$. This quantity is plotted in figure 3 using the values of the constants $\lambda=11^{\circ}, A=1000, B=0,10,30,1000$. 
The curves in figure 3 may be used for values of $A$ other than 1000. If the desired value of $A=a$, then the spread read from the curves must be multiplied by $\sqrt{ }\{1000 / a\}$ while $B$ is now the ratio $1000 B / a$.

The same spread is obtained for places the same distance from the transmitting antenna but off the main axis of the beam. The maximum of the incoming directions is, however, then no longer along the great circle connecting the transmitter to the receiver (except in one special case treated later on).

A noticeable feature of the curves in figure 3 is that, at an angular distance of about $255^{\circ}$ from the transmitter, the spread of the incoming directions is apparently independent of the beam width of the transmitting antenna and thus depends only on the reflection scattering: coefficient.

The value of the standard deviation of the spread at this particular distance is given by

$$
\sigma=\frac{1}{2} \sqrt{\frac{n-1}{A}} \text {, where } \mathrm{n}=\text { number of steps. }
$$

For $\lambda=11^{\circ}, n=23$ at $253^{\circ}$, so that $\sigma \approx 4 .^{\circ} 2(A=1000)$.

The vicinity of $\Lambda=255^{\circ}$ has further interesting properties, as will be seen in the next section.

At the antipodal point $\left(\Lambda=180^{\circ}\right)$ the value of $N^{\prime}$ can be found, by summing the series in (25), to be given by $N^{\prime}=\frac{A B}{A+\frac{n-2}{2} B}$.

Since the standard deviation of the spread of power in the transmitter beam is given by

$$
\sigma=\frac{1}{\sqrt{2 B}}
$$

the ratio of the spreads of the received fan of waves at the antipodal point to the transmitted fan is given by

$$
\frac{\sigma(R)}{\sigma(T)}=\sqrt{1+\overline{n-2} \frac{B !}{2 A}}
$$

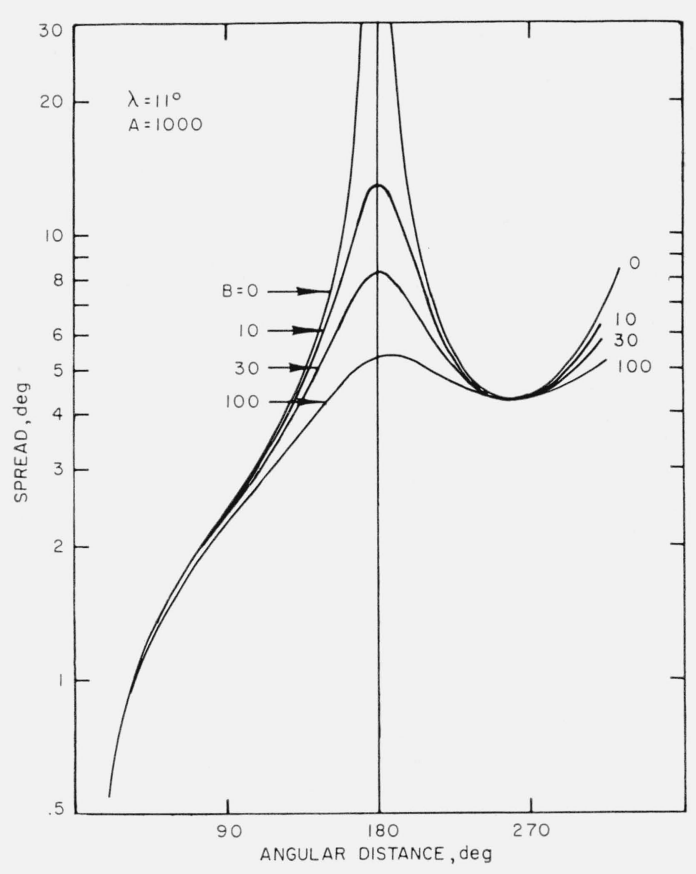

Figure 3. Spread of incoming directions as a function of the distance from the transmitter for various transmitting antenna beamwidths.

The half-power beam-width of the transmitting antenna is $\pm \frac{48}{\sqrt{B}} \mathrm{deg}$. 


\section{Deviation of Incoming Directions From the Great Circle Direction}

The maximum of (14) is obtained at

$$
\xi_{\max }=\frac{M^{\prime}}{2 N^{\prime}} \theta
$$

If the receiver is on the main axis of the transmitter beam, $\theta=0$, so that the received directions have a maximum along the great circle to the transmitter. Off the main axis, the ratio of the deviation of the maximum of the received directions to the angle between the transmitter beaming direction and the great circle from the transmitter to the receiver is given by

$$
\frac{\xi_{\max }}{\theta_{T}}=\frac{M^{\prime}}{2 N^{\prime}} .
$$

This quantity is plotted in figure 4 .

At the antipodal point to the transmitter, the received angle follows the transmitted angle exactly, as would be expected. There is a further interesting region, distant about $257^{\circ}$ from the transmitter, where the received bearings are always clustered about the great circle direction irrespective of the bearing direction and beam width of the transmitter and independent of the scattering coefficient $A$. From the expressions for $M^{\prime}$ and $N^{\prime}$ in (24) and (25) it can be seen that (31) goes to zero when the bracketed term in (24) goes to zero. This term does not contain $A$ or $B$.

The line at about $255^{\circ}$ from the transmitter where the spread is independent of the transmitting antenna beam width and where the mean direction of the incoming waves is always along the great circle direction, could perhaps be called the "reciprocal line" to the transmitter. These conditions hold for an ionosphere which is concentric with the earth and in which all paths are possible. It is obvious that experimental measurements taken at this distance offer a unique opportunity to differentiate between scattering effects and other ionospheric effects.

The sign of the variation of $\xi_{\max }$ with $\theta_{T}$ is important. From figure 1 , if the receiver is at $W$ with the transmitter beam aimed along the line $T R$, then $\theta$, the longitude of $W$ with respect to $T R$ is positive and $\xi_{3}$, the deviation, is positive when measured in the direction shown. At receiving points more than $180^{\circ}$ distant from the transmitter, $\xi_{\max }$ must be measured in a clockwise direction from the great circle (long path) connecting the receiver to the transmitter.

The reciprocal line effect discussed above may be difficult to observe since the short path to the transmitter is only about $100^{\circ}$ of angular distance long. Backward radiation will then, of course, lead to the bearing deviations expected at $Y$ in figure 4.

Figure 4. Ratio of the deviation of the direction of the maximum of the incoming fan of rays from the great circle direction to the angle between the transmitter beam and the great circle direction to the receiver.

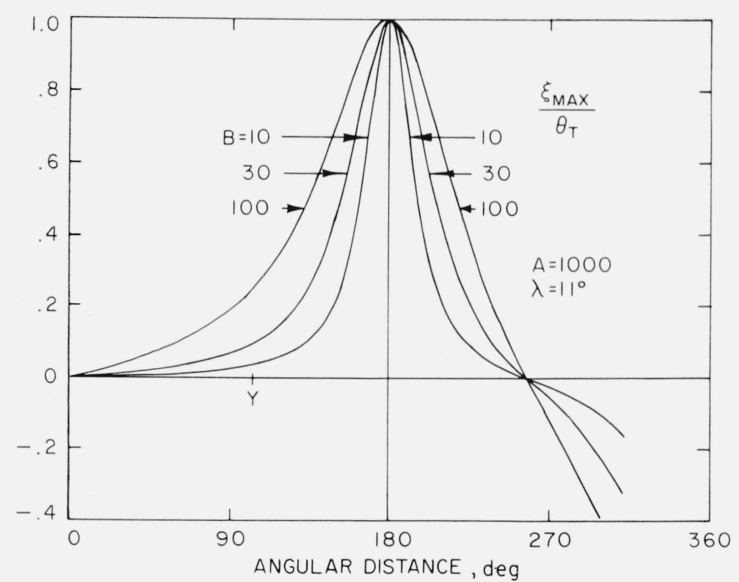




\section{Effects of an Absorbing Region}

The presence of a region in the ionosphere which inhibits the propagation of radio waves (for example, the absorbing regions near the auroral zones or a region of low critical frequency) may be introduced into the analysis by a suitable modification of the distribution along any line $\Lambda$.

Let the undisturbed distribution of signals emergent from the line $\Lambda_{n}$ be given, as before, by the expression

$$
P\left(\Lambda_{n}\right) \propto \exp -\left(L \theta_{n}^{2}-M \theta_{n} \phi_{n}+N \phi_{n}^{2}\right) .
$$

Consider an absorbing region placed as shown at $E$ in figure 5a. This is along one of the latitude lines in the coordinate system referred to $T$ as a pole. Then the shaded areas are the shadow zones as far as great circle propagation is concerned; $A P$ is the point antipodal to the transmitter. The edge $E$ is at $\theta=0$, i.e., at the center of the main beam of the transmitting antenna.

Then

$$
P^{\prime}\left(\Lambda_{n+1}\right) \propto \exp -\left(L^{\prime} \theta_{n+1}^{2}-M^{\prime} \theta_{n+1} \xi_{n+1}+N^{\prime} \xi_{n+1}^{2}\right) \text { with } \xi \text { now limited to the values } \xi>\frac{S_{n}}{S} \theta_{n+1} .
$$

Thus

$$
\begin{aligned}
P\left(\Lambda_{n+1}\right) \propto \int_{-\frac{S_{n}}{S} \theta}^{\infty} \exp -A(\phi-\xi)^{2} \cdot P^{\prime}\left(\Lambda_{n+1}\right) \cdot d \xi & \propto \exp -\left(L \theta_{n+1}^{2}-M \theta_{n+1} \phi_{n+1}\right. \\
& \left.+N \phi_{n+1}^{2}\right) \cdot\left[1+\operatorname{erf}\left\{\sqrt{A+N^{\prime}}\left(\frac{S_{n} \theta}{S}-\frac{2 A \phi+M^{\prime} \theta}{2\left(A+N^{\prime}\right)}\right)\right\}\right] .
\end{aligned}
$$

Since we are mainly interested in regions well within the shadow of the stop, we may replace the error function by its approximation

$$
\operatorname{erf}(v) \approx 1-\frac{\exp (-v)^{2}}{v} .
$$

This gives, for $v$ greater than zero, i.e., within the shadow area,

$$
P\left(\Lambda_{n+1}\right) \propto \exp -\left(L \theta^{2}-M \theta \phi+N \phi^{2}\right) \cdot[1+\operatorname{erf}(v)] \approx 2 \exp -\left(L \theta^{2}-M \theta \phi+N \phi^{2}\right) .
$$

(a)

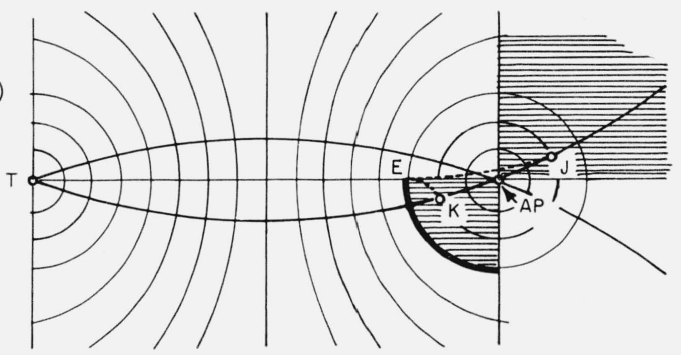

(b)

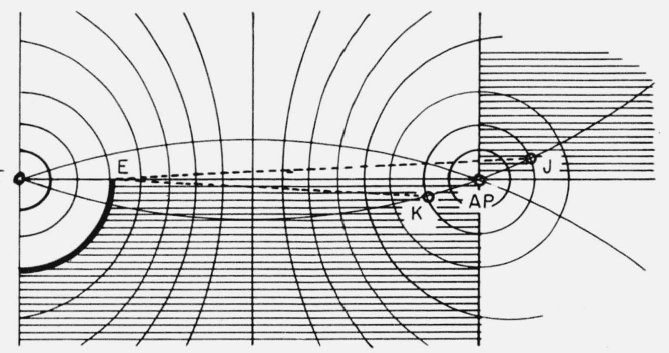

Figure 5. Shadow zones created by an opaque stop. 
The exponential term represents a fan of rays centered on the edge of the stop, with the same spread as the incoming beam. The situation can be represented physically as in figure 6 .

The distribution emergent from the line $\Lambda_{n}$ is given by (32), and the distribution incident on $\Lambda_{n+1}$ is given by (33), provided that $\xi>\frac{S_{n}}{S} \theta$. For any $\theta$, the maximum value of the incident distribution is given when $\xi=\frac{S_{n}}{S} \theta$, i.e., from (33),

$$
\begin{aligned}
P^{\prime}\left(\Lambda_{n+1}\right) \propto \exp -\left\{L^{\prime}-\frac{S_{n}}{S} M^{\prime}+\left(\frac{S_{n}}{S}\right)^{2} N^{\prime}\right\} \theta^{2} & \\
& =\exp -N\left(\frac{S_{n+1}}{S}\right)^{2} \theta^{2} \text {, where } N \text { is the factor in } P\left(\Lambda_{n}\right)
\end{aligned}
$$

For any $\theta, P^{\prime}\left(\Lambda_{n+1}\right)$ given by (33) yields the same spread for $\xi$ so that typical plots for $P^{\prime}\left(\Lambda_{n+1}\right)$ at different positions within the shadow zone are as shown in figure 6a. This spread of $\xi$, since it is independent of $\theta$, is obtained by putting $\theta=0$ in (33), i.e., $P^{\prime}\left(\Lambda_{n+1}\right) \propto \exp -N^{\prime} \xi^{2}$

$$
=\exp -N^{\prime}\left(\frac{S_{n}}{S}\right)^{2} \theta^{2} \text { when } \xi \text { is written in terms of } \theta \text {. }
$$

From (35), $P^{\prime}\left(\Lambda_{n+1}\right) \quad \propto \exp -N_{n}\left(\frac{S_{n+1}}{S}\right)^{2} \theta^{2}$, for the value of $P^{\prime}(\xi)_{\max }$

$$
=\exp -N_{n}\left(\frac{S_{n+1}}{S_{n}}\right)^{2} \xi^{2}, \text { in terms of } \xi
$$

On emerging from the scattering line $\Lambda_{n+1}$, the distributions shown in figure 6a are broadened by the scattering factor $A$. Typical shapes would then be as in figure $6 \mathrm{~b}$. This is in agreement with the expression for $P\left(\Lambda_{n+1}\right)$ given in (34). Thus, for receiving points within the shadow of the stop, we may replace the transmitter by a new source at the edge of the stop with its polar diagram alined in the direction of the great circle connecting the transmitter to the stop edge. The required virtual source at $E$ has a polar diagram of the form

$$
P(\phi) \propto \exp -N \phi^{2} \text {. }
$$

The analysis given so far in this section certainly applies to receiving points within the first shadow zone of the stop, i.e., places like $K$ in figures $5 \mathrm{a}$ and $5 \mathrm{~b}$. The situation at places like $J$ beyond the antipodal point to the transmitter $(A P)$ may be slightly different. Provided the distance $E J$ is less than $180^{\circ}$, the angle which the great circle through $E$ and $J$ makes

Figure 6. Distributions of directions near the edge of an opaque stop.
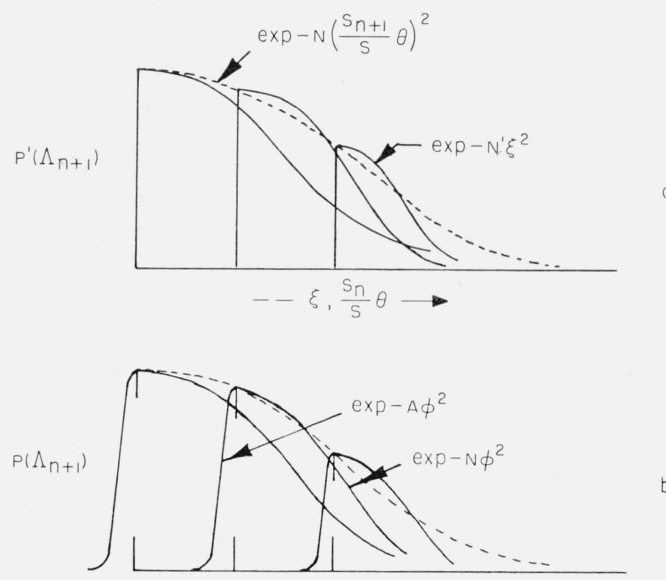
with the line $T-E-A P$ is such that the required initial scattering direction is away from the immediate shadow zone. In this case the beam-width of the source at the shadow edge is given by

$$
P(\phi) \propto \exp -A \phi^{2} .
$$

These correspond to the very narrow beams shown in the left-hand parts of the distributions sketched in figure 6.

This result may be obtained directly from (33) since, under these conditions we obtain

$$
\begin{aligned}
& P\left(\Lambda_{n+1}\right) \propto \exp -\left(L \theta_{n+1}^{2}-M \theta_{n+1} \phi_{\mathrm{n}+1}+N \phi_{n+1}^{2}\right) \cdot[1-\operatorname{erf}(v)] \\
& \quad \propto \frac{1}{\left[2\left(A+N^{\prime}\right) \frac{S_{n}}{S}-M^{\prime}\right] \theta-2 A \phi} \cdot \exp -\left\{\left(L^{\prime}+\overline{A+N^{\prime}}\left(\frac{S_{n}}{S}\right)^{2}-M^{\prime} \frac{S_{n}}{S}\right) \theta^{2}-2 A \frac{S_{n}}{S} \theta \phi+A \phi^{2}\right\}
\end{aligned}
$$

$\propto \exp -A \phi^{2}$, for large $\phi$.

\section{Conclusion}

The theory presented above is in agreement with many of the observational results which have been published previously [Whale, 1956; Whale, 1959; Whale, 1962]. It leads to a much better appreciation of the characteristics of the antipodal area than has been possible heretofore and suggests interesting phenomena occurring at a distance of about $255^{\circ}$ from the transmitter.

Experimental investigations of the phenomena described above are being conducted; the results will be submitted to this journal.

\section{References}

Whale, H. A. (1956), An estimate of the size of the antipodal area in shortwave radio propagation, J. Atmospheric Terrest. Phys. 9, 159-161.

Whale, H. A. (1959), The effects of ionospheric irregularities and the auroral zone on the bearings of shortwave radio signals, J. Atmospheric Terrest. Phys. 13, 258-270.

Whale, H. A. (1962), Ionospheric irregularities and long-distance radio propagation, J. Res. NBS 66D (Radio Prop.) No. 3, 265-272.

(Paper 67D3-263) 